\title{
Heartbeat: Focus on valvular heart disease
}

We have seen a recent upsurge in research related to valvular heart disease due to increasing recognition of the prevalence and adverse outcomes associated with heart valve disease, improved imaging modalities allowing early diagnosis and sequential evaluation, and new treatment options which offer the promise of reduced symptoms and longer survival in patients with these conditions. Valve disease research includes a spectrum of methodological approaches including basic science to understand disease mechanism and identify potential therapeutic targets, epidemiological studies to estimate prevalence and determine modifiable risk factors, imaging studies to improve diagnosis and quantitation of disease severity, clinical studies to better understand the pathophysiology and disease course, and interventional studies to evaluate the efficacy of surgical and transcatheter valve procedures. In this issue of Heart, we present a few of the recent interesting clinical studies of outcomes in patients with valvular heart disease. These papers highlight the challenges of clinical research in patients with valvular heart disease and suggest a path forward using advanced imaging to study disease mechanisms as well as larger datasets to study clinical outcomes, even in subset of patients with less common types or combinations of valve disease.

The effect of anticoagulation on valve haemodynamics after transcatheter valve replacement (TAVR) was examined in a multicentre study of 2466 patients (46\% men, mean age of 81 years). ${ }^{1}$ A propensity score matched analysis with 622 patients in each group suggested that the absence of treatment with anticoagulation after TAVR was associated with a significant increase in transvalvular gradient over $29 \pm 18$ months of follow-up. In contrast, those receiving anticoagulation had no change in gradient over time and had a lower incidence of haemodynamic deterioration $(0.6 \%$ vs $3.9 \%, \mathrm{p}<0.001)$.

Correspondence to Professor Catherine M Otto, Division of Cardiology, University of Washington, Seattle,WA 98195, USA; cmotto@uw.edu

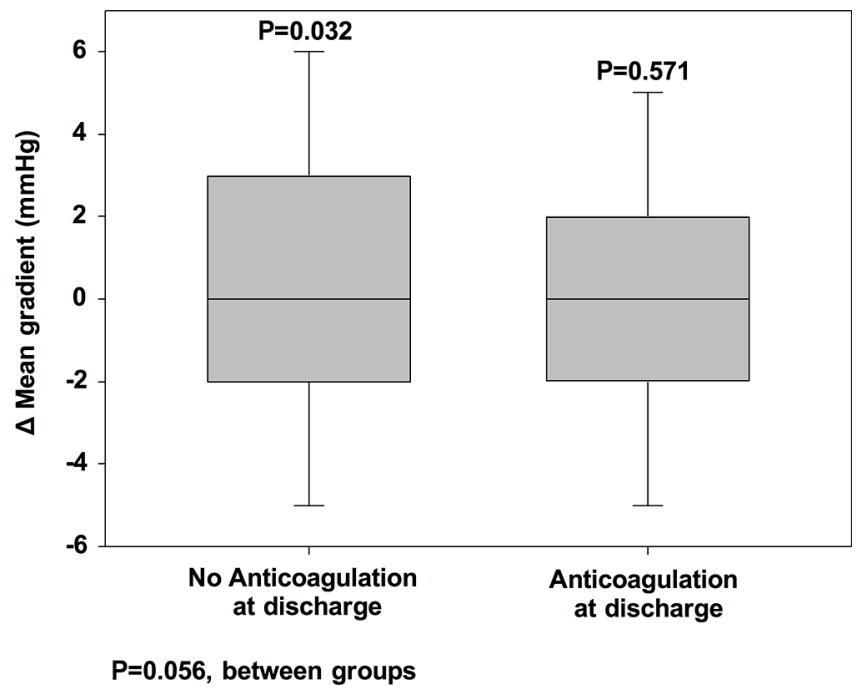

Figure 1 Absolute changes in mean transvalvular gradient between discharge and 1-year follow-up in the propensity-matched population according to the presence of anticoagulation therapy at hospital discharge (analyses conducted on log-transformed data).

However, a transvalvular mean gradient of $10 \mathrm{~mm} \mathrm{Hg}$ or higher was not associated with an increased risk of death or stroke over this follow-up interval (figure 1).

In the accompanying editorial, Doris and Dweck ${ }^{2}$ point out that TAVR now is an established treatment for severe aortic

stenosis with reliable data showing valve durability up to 5 years after valve implantation. However, concerns remain about long-term durability (10-20 years) of transcatheter bioprosthetic valves. Several factors might be associated with limited valve durability including the geometry

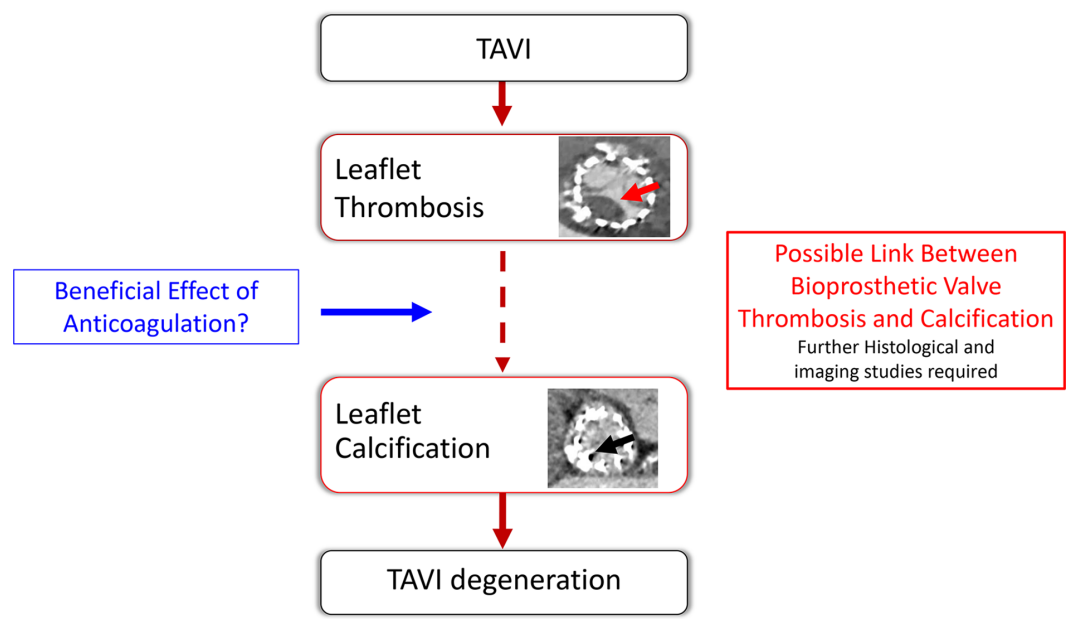

Figure 2 Possible link between bioprosthetic valve thrombosis and calcification. The study by Del Trigo et al suggests that anticoagulation may preserve transcatheter aortic valve implantation (TAVI) valve haemodynamics. The potential mechanism for this observation is not clear. Concern has recently grown regarding the incidence of subclinical leaflet thrombosis (red arrow) in patients undergoing TAVI, and it is hypothesised that this may lead to future calcific degeneration of bioprosthetic TAVI valves (black arrow). Future research should focus on investigating this possible mechanistic link between bioprosthetic leaflet thrombosis and valve calcification/degeneration and the role that anticoagulant therapy might play in improving TAVI valve durability. 


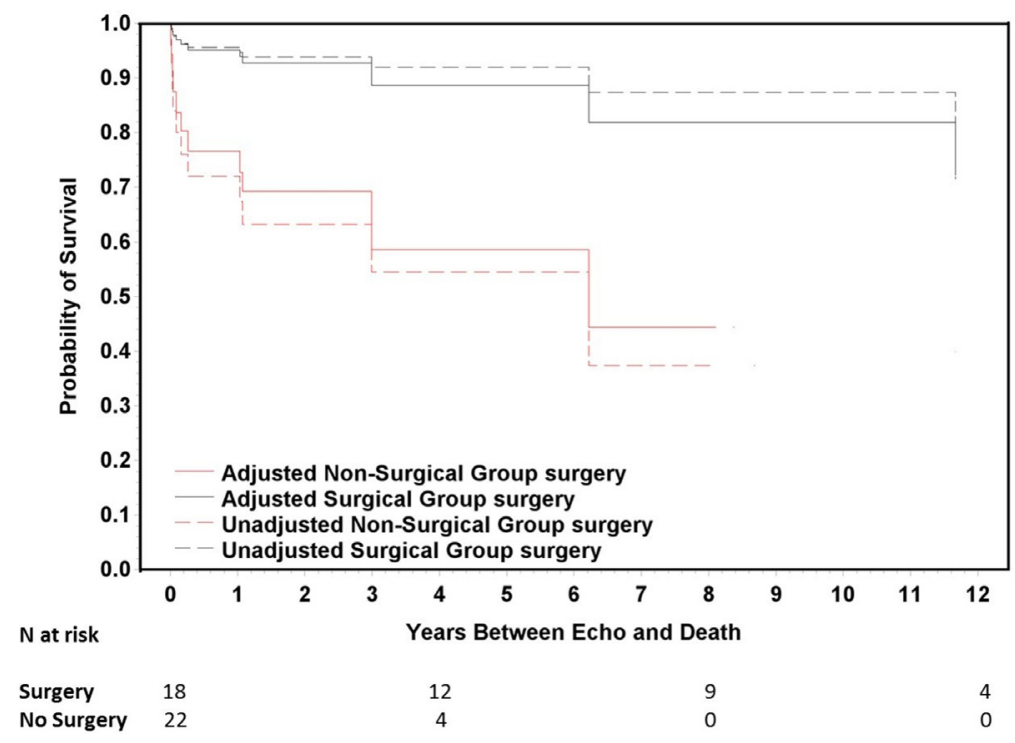

Figure 3 Adjusted and unadjusted survival curves in surgery and non-surgical groups. of valve design, effects of crimping on the leaflet tissue, and suboptimal anticalcification treatments with early generation valves, among other considerations. However, attention have focused recently on the possible role of valve thrombosis as a contributor to reduced long term durability. Doris and Dweck ${ }^{2}$ ponder the possible mechanisms that might link valve thrombosis to subsequent tissue calcification (Figure 2). They conclude that "Research must now focus on gaining a greater understanding of the link between valve thrombosis and degeneration, with the hope that ultimately this will help inform optimal management to prolong bioprosthetic valve longevity and improve patient outcomes." Readers and researchers may be interested in other recent articles in Heart addressing the issue of structural degeneration of TAVR valves. $^{34}$

In patients with chronic severe aortic regurgitation (AR), current guidelines

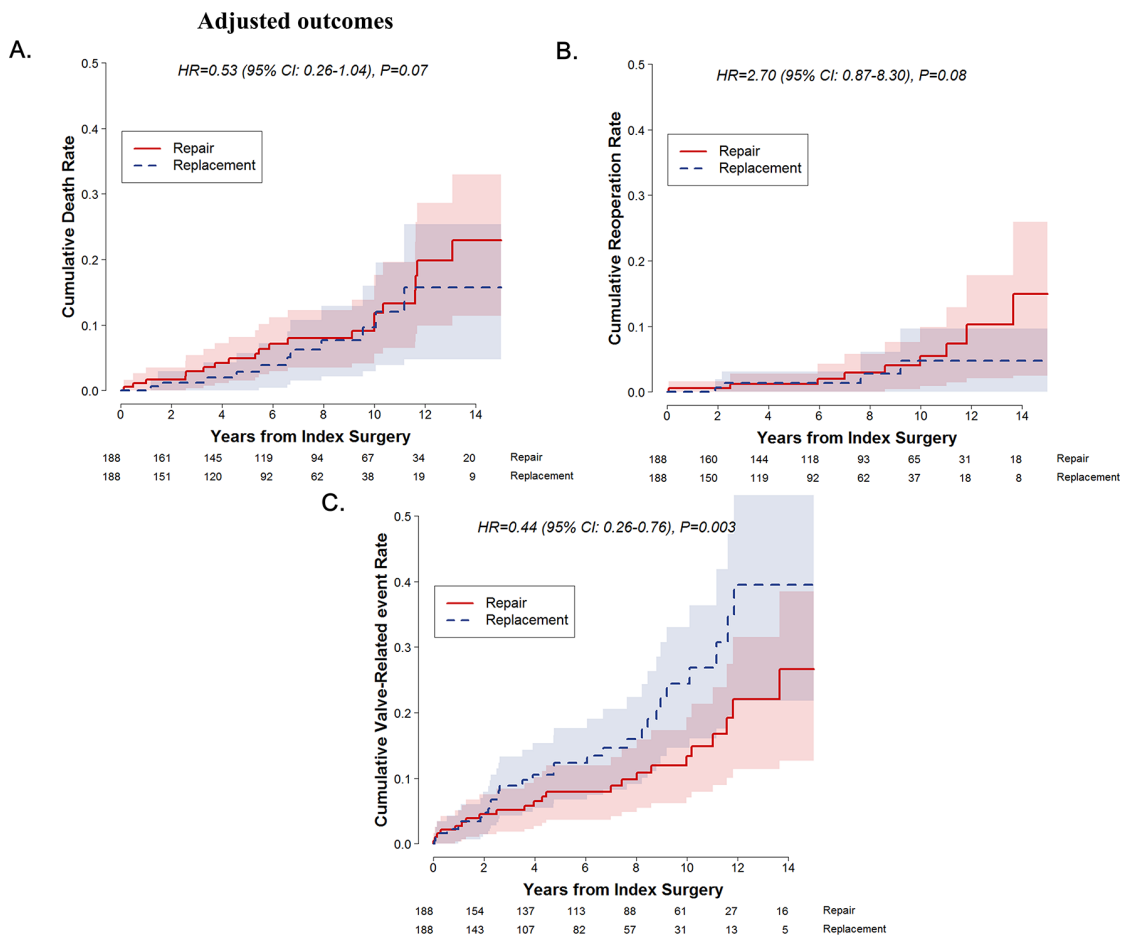

Figure 4 Adjusted Kaplan-Meier plots for cumulative overall mortality (A), reoperation (B) and valve-related complication rates (C) according to the types of surgery. Shaded bands indicate areas within $95 \%$ Cls (red=repair; blue=replacement). recommend aortic valve replacement (AVR) at symptom onset or when there is evidence of early systolic dysfunction, manifested as severe left ventricular (LV) dilation or a fall in ejection fraction, even in asymptomatic patients. In patients who are followed prospectively with appropriately timed intervention, LV size and systolic function normalise rapidly after AVR with excellent long-term outcomes. Unfortunately, some patients only present for medical care with advanced LV systolic dysfunction; management in these patients is controversial due to concerns about surgical risk and persistent LV dysfunction after AVR. In order to address these concerns, Fiedler and colleagues queried a large echocardiographic database and found that only about 5\% of patients with severe AR also had an LV ejection fraction $<35 \%$. $^{5}$ In these 40 patients, mortality at a mean follow-up of 6.6 years was $27.8 \%$ in those who underwent AVR and $91.2 \%$ in those who did not undergo AVR, with multivariable analysis confirming a lower mortality with AVR $(\mathrm{HR}=0.143, \mathrm{p}=0.0490)$ (Figure 3$)$.

This study highlights the challenges in clinical studies of valvular heart disease. Although the number of subjects in this analysis is quite small, the initial cohort of patients undergoing echocardiography was quite large (almost 150 000). Patients were not randomised to AVR or medical therapy so that the effects of patient selection cannot be fully adjusted for; perhaps sicker patients did not undergo AVR, for example, but a randomised study of such a rare condition would be difficult. However, this study suggests that with even larger databases, we can add to the evidence base for decision making in patients with valvular heart disease.

Given the complexities and many uncertainties in management of valve disease patients, McConkey et $a l^{6}$ 'strongly endorse the concept of Heart Valve Centres-specialist centres resourced with an array of imaging modalities, access to percutaneous and surgical valve treatments, and high-level expertise in valvular heart disease and key related cardiac and non-cardiac disciplines. These centres can provide a network of dedicated valve clinics with access to clinical expertise and consistent high-quality imaging to facilitate careful follow-up and timely referral to a dedicated Heart Team for consideration of surgery or percutaneous intervention.'

Most studies on outcomes after surgical intervention for mitral valve disease predominantly included patients with myxomatous (eg, degenerative) mitral 


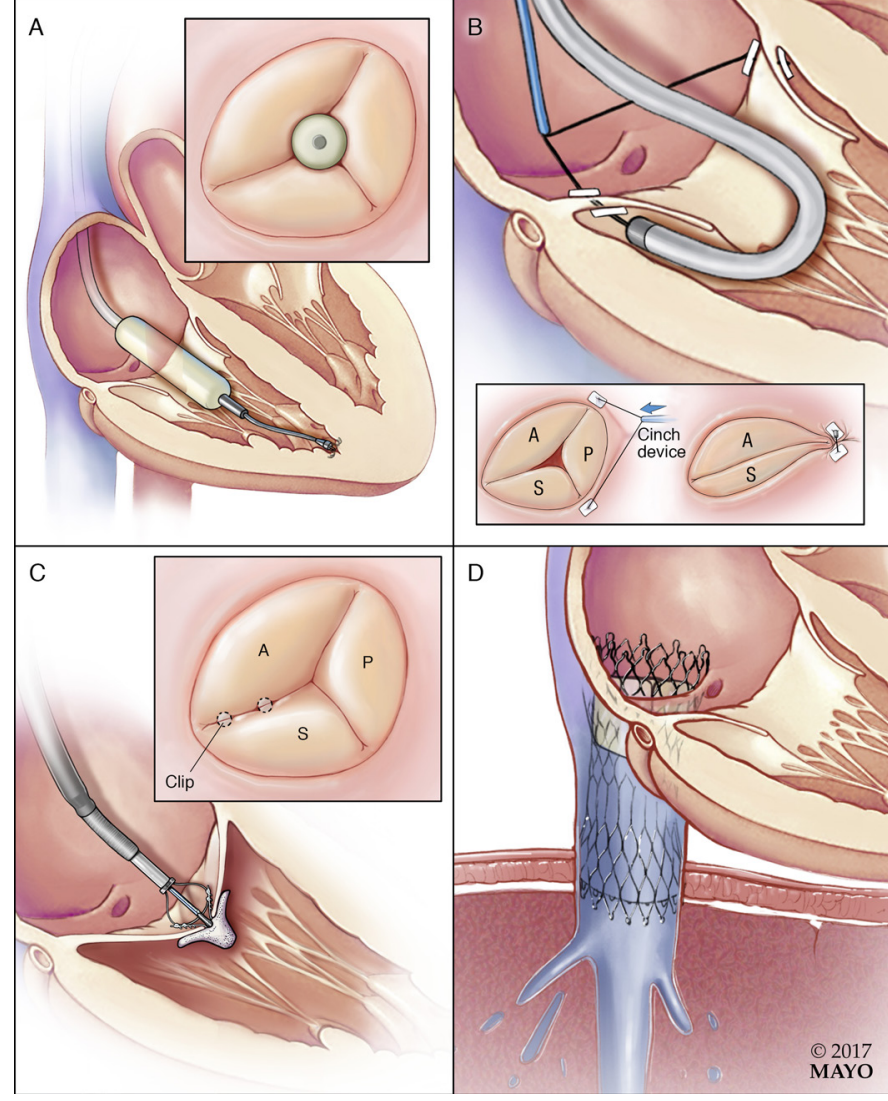

Figure 5 Multiple percutaneous devices are in development for the treatment of tricuspid regurgitation. Panel $(A)$ is the FORMA device, a tricuspid spacer which occupies the regurgitant orifice and provides a surface against which coaptation can occur. Panel (B) demonstrates the TriAlign, which percutaneously reproduces a surgical Kay bicuspidisation. Panel (C) shows the MitraClip being used in the tricuspid position. Panel (D) demonstrates a stented caval valve implanted in the inferior vena cava.

regurgitation, although rheumatic mitral valve disease is much more common on a worldwide basis. In this issue of Heart, Kim and colleagues ${ }^{7}$ report clinical outcomes in 1731 patients (69\% women, mean age 52 years) undergoing mitral valve surgery for rheumatic valve disease. In a propensity matched analysis, there was no difference in mortality or re-reoperation rates for repair versus replacement but there were fewer valve-related complications in those undergoing valve repair (HR, 0.57 ; 95\% CI 0.33 to 0.99 ), primarily due to a lower risk of haemorrhagic events (figure 4).

Antunes ${ }^{8}$ suggests that the major issue with valve repair for rheumatic disease is 'the increased need for reoperation for progressive fibrosis and distortion of the valve caused by the progression or recurrence of the rheumatic process'. However, 'not all rheumatic populations are equal. A better knowledge of the rheumatic valve pathology and the evolution of repair techniques have contributed to improved results. Hence, valve repair, however challenging, is still worthwhile and the percentage of valves repaired increases with the experience of the surgeon and the will to preserve the valve. It is vital that all surgeons dealing with this type of pathology gain adequate experience and overcome the unavoidable learning curve, which can only be obtained by exposure to enough patients with this condition.'

Also, in this focus issue on valvular heart disease, you will find two excellent review articles on outcomes and management of isolated tricuspid valve regurgitation (figure 5 ) $^{9}$ and on the clinical utility of stress echocardiography in patients with valvular heart disease. ${ }^{10}$
The Education in Heart article in this issue discusses minimally invasive mitral valve repair. ${ }^{11} \mathrm{Be}$ sure to try the Image Challenge question. ${ }^{12}$ Hint: it is about valve disease too!

Competing interests None declared.

Patient consent Not required.

Provenance and peer review Commissioned; internally peer reviewed.

(C) Article author(s) (or their employer(s) unless otherwise stated in the text of the article) 2018. All rights reserved. No commercial use is permitted unless otherwise expressly granted.

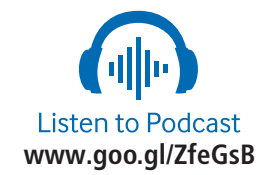

D Check for updates

To cite Otto CM. Heart 2018;104:789-791.

Heart 2018;104:789-791.

doi:10.1136/heartjnl-2018-313376

\section{REFERENCES}

1 Del Trigo M, Muñoz-García AJ, Latib A, et al. Impact of anticoagulation therapy on valve haemodynamic deterioration following transcatheter aortic valve replacement. Heart 2018;104:814-20.

2 Doris MK, Dweck MR. Is bioprosthetic leaflet thrombosis a trigger to valve degeneration? Heart 2018:104:792-3.

3 Foroutan F, Guyatt GH, Otto CM, et al. Structural valve deterioration after transcatheter aortic valve implantation. Heart 2017;103:1899-905.

4 Bagur R, Pibarot P, Otto CM. Importance of the valve durability-life expectancy ratio in selection of a prosthetic aortic valve. Heart 2017;103:1756-9.

5 Fiedler AG, Bhambhani V, Laikhter E, et al. Aortic valve replacement associated with survival in severe regurgitation and low ejection fraction. Heart 2018;104:835-40.

6 McConkey HZ, Rajani R, Prendergast BD. Improving outcomes in chronic aortic regurgitation: timely diagnosis, access to specialist assessment and earlier surgery. Heart 2018;104:794-5.

7 Kim WK, Kim HJ, Kim JB, et al. Clinical outcomes in 1731 patients undergoing mitral valve surgery for rheumatic valve disease. Heart 2018;104:841-8.

8 Antunes MJ. Repair for rheumatic mitral valve disease. The controversy goes on!. Heart 2018;104:796-7.

9 Fender EA, Zack CJ, Nishimura RA. Isolated tricuspid regurgitation: outcomes and therapeutic interventions. Heart 2018;104:798-806.

10 Lancellotti P, Dulgheru R, Go YY, et al. Stress echocardiography in patients with native valvular heart disease. Heart 2018;104:807-13

11 Javadikasgari H, Suri RM, Tappuni B, et al. Minimally invasive mitral valve repair. Heart 2018;104:861-7.

12 Obayashi Y, Izumi C, Nakagawa Y. Man in his 50s with chest pain and dyspnoea. Heart 2018;104:840. 